\title{
Pinosylvin inhibits migration and invasion of nasopharyngeal carcinoma cancer cells via regulation of epithelial-mesenchymal transition and inhibition of MMP-2
}

\author{
YI-CHING CHUANG $^{1 *}$, MING-CHANG HSIEH ${ }^{2,3^{*}}$, CHIA-CHIEH LIN $^{1}$, \\ YU-SHENG LO ${ }^{1}$, HSIN-YU HO ${ }^{1}$, MING-JU HSIEH ${ }^{1,4-6}$ and JEN-TSUN LIN ${ }^{6-8}$ \\ ${ }^{1}$ Oral Cancer Research Center, Changhua Christian Hospital, Changhua 500; \\ ${ }^{2}$ School of Medical Laboratory and Biotechnology, Chung Shan Medical University; \\ ${ }^{3}$ Department of Clinical Laboratory, Chung Shan Medical University Hospital; ${ }^{4}$ Institute of Medicine, \\ Chung Shan Medical University, Taichung $40201 ;{ }^{5}$ Graduate Institute of Biomedical Sciences, \\ China Medical University, Taichung 404; ${ }^{6}$ Post Baccalaureate Medicine, National Chung Hsing University, Taichung 40201; \\ ${ }^{7}$ Division of Hematology and Oncology, Department of Medicine, Changhua Christian Hospital, Changhua 500; \\ ${ }^{8}$ School of Medicine, Chung Shan Medical University, Taichung 40201, Taiwan, R.O.C.
}

Received March 18, 2021; Accepted May 5, 2021

DOI: $10.3892 / o r .2021 .8094$

\begin{abstract}
Nasopharyngeal carcinoma (NPC) is a tumor located in the nasopharynx with highly invasive and metastatic properties. Metastasis is a primary cause of mortality in patients with NPC. The terpenoid polyphenol pinosylvin is a known functional compound of the Pinus species that exhibits anti-inflammatory effects; however, the effect of pinosylvin on human NPC cell migration and invasion is unclear. The present study aimed to investigate the functional role of pinosylvin in NPC cells (NPC-039, NPC-BM and RPMI 2650). Gap closure and Transwell assay indicated that pinosylvin at increasing concentrations inhibited migration and invasion of NPC-039 and NPC-BM cells. In addition to inhibiting the enzyme activity of MMP-2, pinosylvin also decreased the protein expression levels of MMP-2 and MMP-9. Pinosylvin decreased the expression of vimentin and $\mathrm{N}$-cadherin and
\end{abstract}

Correspondence to: Dr Ming-Ju Hsieh, Oral Cancer Research Center, Changhua Christian Hospital, 135 Nanxiao Street, Changhua, Changhua 500, Taiwan, R.O.C.

E-mail:170780@cch.org.tw

Dr Jen-Tsun Lin, Division of Hematology and Oncology, Department of Medicine, Changhua Christian Hospital, 135, Nanxiao Street, Changhua, Changhua 500, Taiwan, R.O.C.

E-mail: 111227@cch.org.tw

${ }^{*}$ Contributed equally

Abbreviation: NPC, nasopharyngeal carcinoma

Key words: pinosylvin, nasopharyngeal cancer, matrix metalloproteinase, epithelial-mesenchymal transition, MAPK significantly increased the expression of zonula occludens-1 and E-cadherin in NPC cells. Additionally, pinosylvin suppressed the invasion and migration ability of NPC-039 and NPC-BM cells by mediating the p38, ERK1/2 and JNK1/2 pathways. The present results revealed that pinosylvin inhibited migration and invasion in NPC cells.

\section{Introduction}

The terpenoid polyphenol pinosylvin (trans-3,5-dihydroxystilbene) is a stilbene present in the heartwood of coniferous trees of the genus Pinus (1). Many studies have demonstrated that biological characteristics of pinosylvin include antibacterial and antifungal activity (2) and protection against oxidative stress in human cells (3). Pinosylvin regulates Src/ERK and GSK-3/ $\beta$-catenin signaling to inhibit tumor cell growth (4). Pinosylvin has been shown to inhibit the expression of MMP-2 and MMP-9 in human fibrosarcoma HT1080 cells (5).

Nasopharyngeal carcinoma (NPC) is a tumor located in the nasopharynx and is caused by epithelial cells covering the nasopharyngeal surface. Unlike other head and neck epithelial cancers, NPC is highly invasive and metastatic (6). NPC is particularly prevalent in Southern China, Southeast Asia, North Africa and the Arctic region, which is a unique geographical distribution (7). Four primary causes of nasopharyngeal carcinoma have been identified, including Epstein-Barr and human papillomavirus infection, genetic susceptibility and consumption of salted fish (8). NPC occurs adjacent to cervical lymph nodes, which increases the risk of metastasis in other parts of the body, thereby causing difficulties in surgical treatment (8). Currently, chemotherapy and radiotherapy can improve the survival rate of patients with advanced NPC (9). Preventing distant metastasis is key to treatment, and more effective systemic drugs should be investigated (10). 
The metastasis of NPC occurs in two stages: Translocation to distant tissue and colonization (11). The initial step degrades and penetrates the extracellular matrix of surrounding tissue (12). Among the involved proteolytic enzymes, zinc-dependent MMPs contribute substantially to proteolytic degradation and intercellular interaction damage (13). Research has indicated that MMP-2 and MMP-9 are key treatment targets for regulation of tumor metastasis in NPC (14), cervical cancer (15) and retinoblastoma (16). Lyu et al (17) reported that liposome-containing thermosensitive liposomes can deliver MMP inhibitors, decreasing the activity of MMP-2 and MMP-9 by 50 and 43\%, respectively, to inhibit metastasis and angiogenesis. Huang et al (18) demonstrated that exosomes with low expression levels of microRNA-34c-3p affect expression of integrin $\alpha 2 \beta 1$ and promote the invasion and migration of non-small cell lung cancer cells.

Epithelial-mesenchymal transition (EMT) is a key process involved in tumor metastasis and recurrence $(19,20)$. Research has indicated that the expression of mesenchymal markers, such as vimentin and $\mathrm{N}$-cadherin, increases during EMT, whereas epithelial marker E-cadherin, a powerful tumor cell invasion inhibitor, is downregulated $(21,22)$. The MAPK pathway is an important intracellular signal transduction pathway that serves a key role in regulating tumor metastasis, as well as regulating cell proliferation, differentiation, apoptosis and angiogenesis (23). The ERK subfamily (typical ERK 1/2/5 and atypical ERK 3/4/7/8) of proteins is known for its contributions to EMT $(23,24)$. PI3K/AKT and MAPK pathways contribute to TGF- $\beta 2$-induced upregulation of Jagged-1, which mimics TGF- $\beta 2$-induced EMT in retinal pigment epithelium cells (25). TGF- $\beta$, in addition to its role in cell differentiation, migration and adhesion, also induces EMT via both Smad and MAPK pathways (26). A previous study indicated that pinosylvin exerts antimetastatic effects on human oral cancer cells (27). However, the antimigratory effect of pinosylvin on NPC cells remains unknown. Therefore, the present study investigated the effect of pinosylvin on NPC cell metastasis and regulation of its signaling.

\section{Materials and methods}

Chemicals. Pinosylvin ( $\geq 97 \%$ purity) was purchased from ChemFaces. DMSO was used to prepare $100 \mathrm{mM}$ storage solution of pinosylvin, which was stored at $-20^{\circ} \mathrm{C}$. The maximum concentration of DMSO used for treatment in medium was $<0.2 \%$. MTT, ERK1/2, p38 and JNK1/2 specific inhibitors (U0126, SB203580 and SP600125) were obtained from Sigma-Aldrich (Merck KGaA).

Cell culture. Nasal cavity cancer cells (RPMI 2650) were obtained from Japanese Collection of Research Bioresources Cell Bank (Osaka, Japan). Human nasopharyngeal cancer cell lines (NPC-039 and NPC-BM) were provided by Dr Jen-Tsun Lin, Department of Hematology and Oncology, Changhua Christian Hospital (Changhua, Taiwan). RPMI-2650 cells were cultured in Eagle's Minimum Essential Medium (Gibco; Thermo Fisher Scientific, Inc.); NPC cell lines were cultured in RPMI-1640 medium (Gibco; Thermo Fisher Scientific, Inc.). All culture media were supplemented with $10 \%$ fetal bovine serum (Gibco; Thermo Fisher Scientific, Inc.),
$1 \mathrm{mM}$ glutamine, $1 \%$ penicillin/streptomycin $(10,000 \mathrm{U} / \mathrm{ml}$ penicillin and $10 \mathrm{mg} / \mathrm{ml}$ streptomycin), $1.5 \mathrm{~g} / 1$ sodium bicarbonate and $1 \mathrm{mM}$ sodium pyruvate. All cell cultures were maintained at $37^{\circ} \mathrm{C}$ in a humidified atmosphere of $5 \% \mathrm{CO}_{2}$.

In vitro cytotoxicity assay (MTT assay). Cytotoxicity was assessed via MTT $(0.1 \%$,) assay. All cells were cultured in 96-well plates $\left(1 \times 10^{4} /\right.$ well $)$ at $37^{\circ} \mathrm{C}$ in $5 \% \mathrm{CO}_{2}$ overnight. Subsequently, supernatant was removed and cultures were treated with different concentrations of pinosylvin $(0,20,40$ and $80 \mu \mathrm{M})$ at $37^{\circ} \mathrm{C}$ for $24 \mathrm{~h}$. Following treatment, the medium containing pinosylvin was removed and MTT reagent $(1 \mathrm{mg} / \mathrm{ml})$ was added to each well at $37^{\circ} \mathrm{C}$ in $5 \% \mathrm{CO}_{2}$. After $4 \mathrm{~h}$, the supernatant containing MTT reagent was removed and DMSO was added to dissolve the formed blue formazan crystals. Absorbance was measured at $595 \mathrm{~nm}$ using spectrophotometry. A total of three independent experimental replicates was performed.

Gap closure assay. Gap closure assay was used to measure migration of NPC-039 and NPC-BM cells over a certain distance. NPC-039 and NPC-BM cells $\left(3 \times 10^{4}\right)$ were grown onto each side of a culture insert (Ibidi $\mathrm{GmbH}$ ) at $37^{\circ} \mathrm{C}$ overnight. After reaching $90 \%$ confluence, culture inserts were removed and gap closure assay was performed. Cultures were treated with pinosylvin $(0,20,40$ and $80 \mu \mathrm{M})$ in serum-free RPMI-1640 (Gibco; Thermo Fisher Scientific, Inc.) at $37^{\circ} \mathrm{C}$ for $24 \mathrm{~h}$. The cell migration distance was observed and photographed after 0,3 and $6 \mathrm{~h}$. Migration was measured using ImageJ 1.47 version software (National Institutes of Health) and expressed as a percentage using the following formula: (Initial gap width of the experimental group-remaining width of the experimental group)/(initial gap width of the control group-remaining width of the untreated control group) $\mathrm{x} 100$. Images were captured under a light microscope (Lecia $\mathrm{GmbH}$ ). The entire procedure was repeated three times and the values are indicated as mean $\pm \mathrm{SD}$.

Cell migration and invasion assay. NPC-039 and NPC-BM cell migration and invasion assays were performed as described by Yang et al (28). Briefly, NPC cells $\left(3 \times 10^{4}\right)$ were placed on the upper well of a Transwell insert (Greiner Bio-One International $\mathrm{GmbH}$ ) with serum-free medium (RPMI-1640) and 10\% FBS-containing medium (RPMI-1640 medium) (600 $\mu \mathrm{l})$ was added to the lower chamber for $24 \mathrm{~h}$ at $37^{\circ} \mathrm{C}$. For the invasion assay, Matrigel $(25 \mathrm{mg} / 50 \mathrm{ml} ; 60 \mu \mathrm{l}$; BD Biosciences) was coated on the upper Transwell at $37^{\circ} \mathrm{C}$, overnight. Migrated or invaded cells were fixed with $99 \%$ methanol at room temperature for $15 \mathrm{~min}$ and stained with Giemsa (1X) at room temperature for $2 \mathrm{~h}$. Images were captured and number of cells was counted under an optical light microscope (Lecia Germany) at 100x magnification using ImageJ 1.47 version cell count software (National Institutes of Health). A total three fields of view was randomly selected for each concentration. Data are presented as the mean \pm SD $(n=3)$.

Gelatin zymography. Enzyme activity of MMP-2 was analyzed via gelatin zymography. Briefly, after plating NPC-039 and NPC-BM cells $\left(5 \times 10^{4}\right.$ cells/well) in 24 -well plates at $37^{\circ} \mathrm{C}$ for $16 \mathrm{~h}$, cells were treated with different concentrations 
A

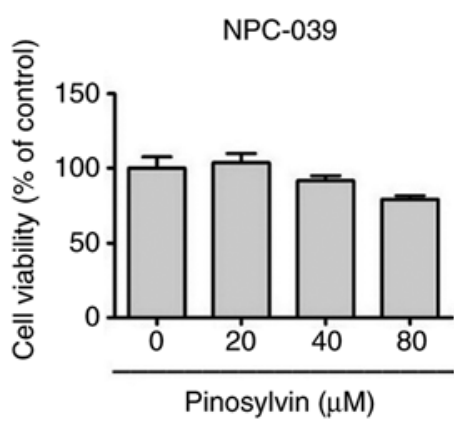

B

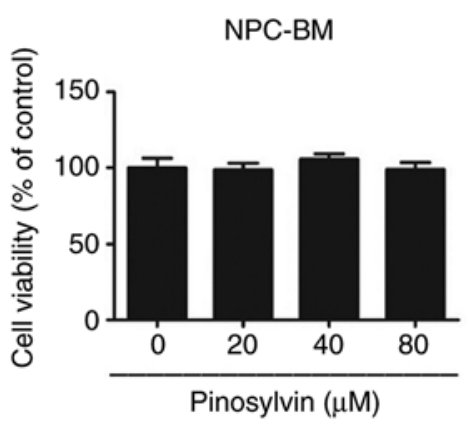

C

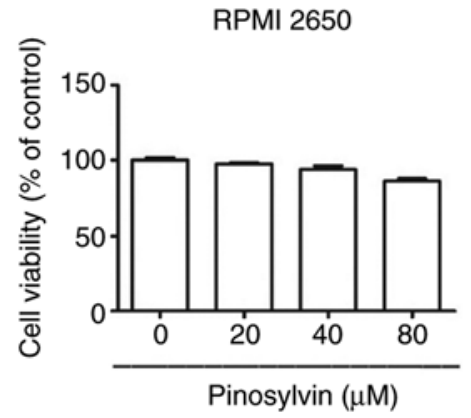

Figure 1. Pinosylvin does not inhibit cell proliferation in three cell lines. (A) NPC-039, (B) NPC-BM and (C) RPMI 2650 cells were treated with pinosylvin $(0,20,40$ and $80 \mu \mathrm{M})$ for $24 \mathrm{~h}$. Cytotoxicity was measured using an MTT assay. Data are presented as the mean \pm SD (n=3). NPC, nasopharyngeal carcinoma.

$(0,20,40$ and $80 \mu \mathrm{M})$ of pinosylvin at $37^{\circ} \mathrm{C}$ for $24 \mathrm{~h}$. Culture medium was collected and subjected to $8 \%$ SDS-PAGE with $0.1 \%$ gelatin as described previously (29).

Western blot analysis. Following treatment with different concentrations of pinosylvin, cells were lysed with 1X RIPA buffer (EMD Millipore) containing protease and phosphatase inhibitor cocktails and subjected to BCA (Thermo Fisher Scientific, Inc.) protein concentration assay. All samples were separated using 10.0 or $12.5 \%$ SDS-PAGE and proteins were transferred onto a PVDF membrane (EMD Millipore). Membranes were blocked with $5 \%$ non-fat milk in TBST $(0.05 \%$ Tween-20) at room temperature for $1 \mathrm{~h}$. Detection was performed with a primary antibody overnight at $4{ }^{\circ} \mathrm{C}$ followed by a horseradish peroxidase (HRP)-conjugated secondary antibody (Anti-rabbit IgG, \#7074, 1:3,000; Anti-mouse IgG, \#7076, 1:3,000, Cell Signaling Technology, Inc.) at room temperature for $1 \mathrm{~h}$. The following antibodies (all 1:1,000; all Cell Signaling Technology, Inc. unless otherwise indicated) were used: Anti-ERK1/2 (cat. no. \#4695; 42, $44 \mathrm{kDa}$ ), anti-JNK1/2 (cat. no. \#9252; 46, $54 \mathrm{kDa}$ ), anti-p38 (cat. no. \#8690; 40 kDa), anti-phosphorylated (phospho-) ERK1/2 (cat. no. \#4370; 42, 44 kDa), anti-phospho-JNK1/2 (cat. no. \#4668; 46, 54 kDa), anti-phospho-p38 (cat. no. \#4511; $43 \mathrm{kDa}$ ), anti-MMP-2 (cat.no.\#87809; $64 \mathrm{kDa}$ ), anti-N-cadherin (cat. no. \#13116; $140 \mathrm{kDa}$ ), anti-E-cadherin (cat. no. \#3195; $135 \mathrm{kDa}$ ), anti-zonula occludens (ZO)-1 (cat. no. \#8193; $220 \mathrm{kDa}$ ), anti-vimentin (cat. no. \#5741; $57 \mathrm{kDa}$ ), anti-MMP-9 (cat. no. \#AB19016; 92 kDa; EMD Millipore) and anti-b-actin (1:5,000; cat. no. NB600-501; 42 kDa; Novus Biologicals). Immunoblotting was observed using HRP chemiluminescent substrates (EMD Millipore). Images were captured using ImageQuant LAS 4000 mini (GE Healthcare) and relative density was quantitated by ImageJ 1.47 version software (National Institutes of Health).

Proteome profiler human protease array. The Proteome Profiler Human Protease Array kit (cat. no. ARY021B; R\&D Systems, Inc.) was used according to the manufacturer's instructions. Array buffer 6 was added into each well of the 4-well Multi-dish and incubated at room temperature for $1 \mathrm{~h}$. Then, $15 \mu \mathrm{l}$ reconstituted protease detection antibody cocktail was added at room temperature for $1 \mathrm{~h}$. Sample mixtures were incubated with membrane overnight at $4^{\circ} \mathrm{C}$. Each membrane was washed with wash buffer for $10 \mathrm{~min}$. Streptavidin-HRP was added into each well and incubated for $30 \mathrm{~min}$ at room temperature. Immunoblotting was observed using HRP chemiluminescent substrate (EMD Millipore). Images were captured using ImageQuant LAS 4000 mini (GE Healthcare) and relative density was quantitated by ImageJ 1.47 version software (National Institutes of Health).

Statistical analysis. The experimental data are expressed as the mean $\pm \mathrm{SD}(\mathrm{n} \geq 3)$. Comparisons between $>2$ groups were analyzed by one-way ANOVA followed by post hoc Tukey's test. Paired student's t-test was used to analyze differences between two groups. All statistical analyses were performed using GraphPad Prism Software Version 5.0 (GraphPad Software, Inc.). $\mathrm{P}<0.05$ was considered to indicate a statistically significant difference.

\section{Results}

Pinosylvin does not induce cytotoxicity in three cell lines. The cytotoxic effects of various concentrations of pinosylvin $(0,20,40$ and $80 \mu \mathrm{M})$ on cell lines were assessed using MTT assay for $24 \mathrm{~h}$ (Fig. 1A-C). Pinosylvin did not exert significant cytotoxic effects on the viability of NPC-039, NPC-BM and RPMI-2650 cell lines. All subsequent experiments examined antimetastatic properties of pinosylvin at non-cytotoxic concentrations.

Pinosylvin inhibits migration and invasion in NPC cell lines. Gap closure assay was performed to assess the effect of pinosylvin on the mobility of NPC cells treated with $0-80 \mu \mathrm{M}$ pinosylvin for 0,3 and $6 \mathrm{~h}$ (Fig. 2). Compared with the control group, the migrated distance of the cell monolayers was significantly decreased at high concentrations $(80 \mu \mathrm{M})$ of pinosylvin. In addition, the effect of pinosylvin on the migration and invasion ability in NPC cells was assessed by Transwell assay (Fig. 3A-D); pinosylvin significantly decreased the migration and invasion abilities of two NPC cell lines.

Pinosylvin changes migration of NPC cell line and inhibits MMP-2 activity. According to the results of Proteome Profiler Human Protease Array (Fig. S1), to lack of observed differences. MMPs regulate cancer cell migration and invasion (13). In order to determine whether MMP-2 and MMP-9 
A Pinosylvin $(\mu \mathrm{M})$

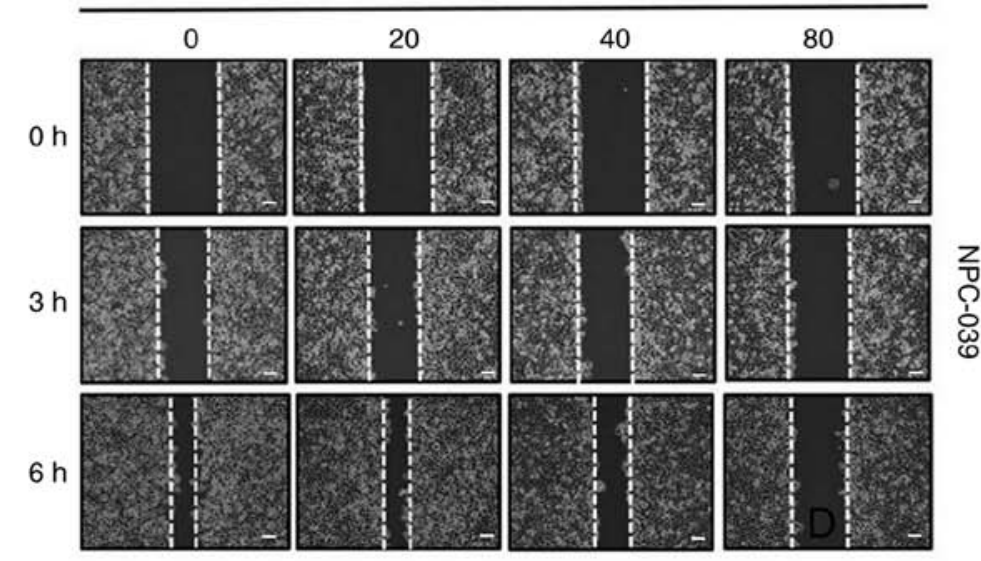

B
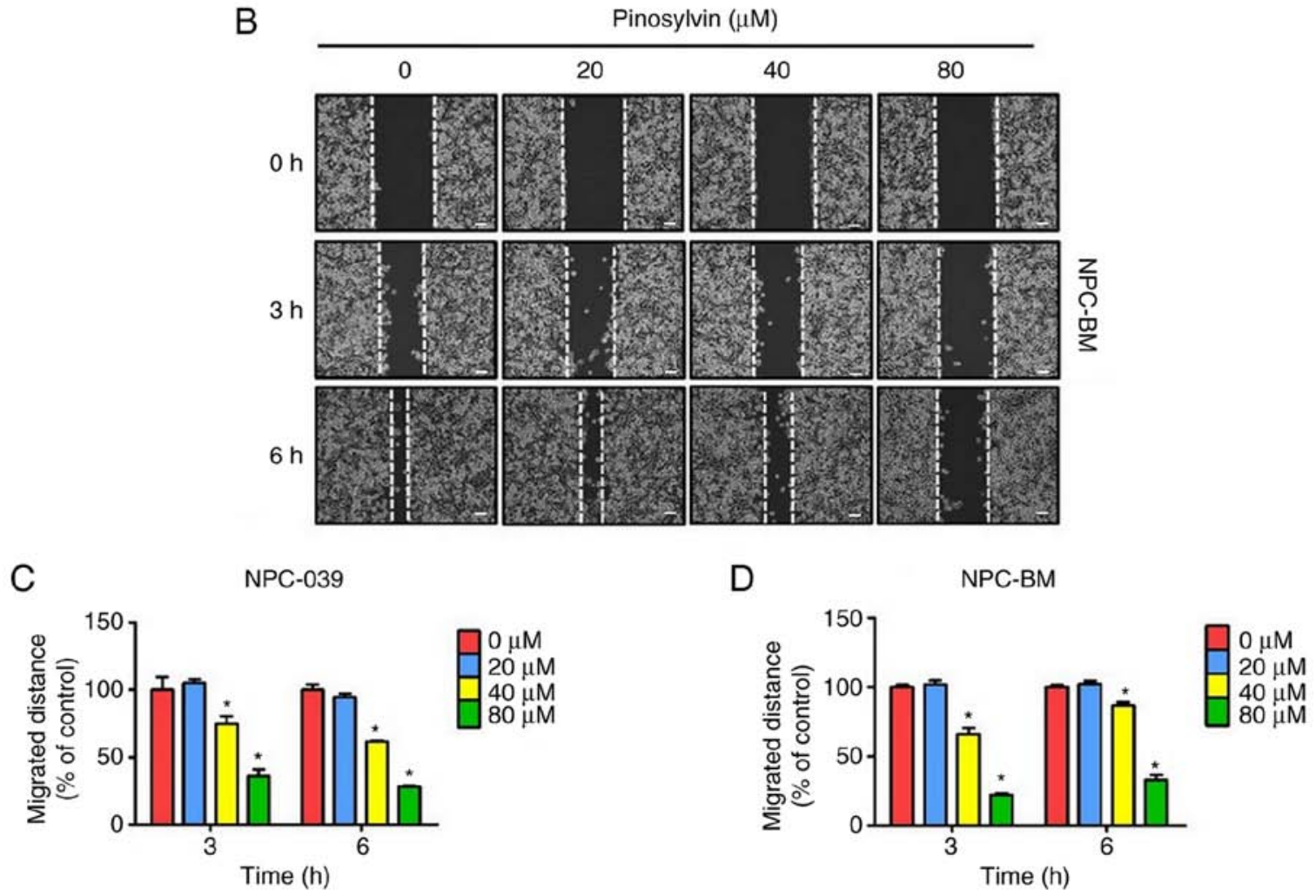

Figure 2. Effect of pinosylvin on gap closure. Cell motility was determined by gap closure assay from 0 and $6 \mathrm{~h}$ in (A) NPC-039 and (B) NPC-BM. Migration of (C) NPC-039 and (D) NPC-BM cells was quantified. Data are presented as the mean \pm SD (n=3). ${ }^{*} \mathrm{P}<0.05$ vs. control. Scale bar, $100 \mu \mathrm{m}$. NPC, nasopharyngeal carcinoma.

are regulated by pinosylvin in two NPC cell lines, zymography and western blotting were performed to analyze enzyme activity and protein concentration. Pinosylvin at the highest concentration significantly decreased enzymatic activity of MMP-2 in two NPC cell lines (Fig. 4A-D). Following $24 \mathrm{~h}$ treatment, a high pinosylvin concentration $(80 \mu \mathrm{M})$ decreased expression levels of MMP-2 and MMP-9 to 54 and 66 in NPC-039 and 52 and $41 \%$ in NPC-BM cells, respectively (Fig. 4E-H).

Pinosylvin affects EMT-associated protein expression in NPC cell lines. When wound healing occurs, organ fibrosis and the initiation of metastasis in cancer progression prompt EMT (30). Analysis of expression levels of EMT-specific proteins (Fig. 5A-D) demonstrated that pinosylvin at a high concentration significantly decreased expression of vimentin and $\mathrm{N}$-cadherin to 58.5 and 62.5 in NPC-039 and 55.0 and $58.5 \%$ in NPC-BM, respectively, and significantly increased expression of ZO-1 and E-cadherin to 80.0 and 101.5 in NPC-039 and 90.0 and $123.0 \%$ in NPC-BM, respectively.

Pinosylvin decreases invasion and migration ability of NPC cell lines via MAPK pathways. Western blotting was performed to detect changes in the molecular mechanisms of MAPK pathways in response to treatment with pinosylvin (Fig. 6A-D). As the concentration of pinosylvin increased, phosphorylation of ERK1/2 and p38 decreased significantly. According to ImageJ analysis of blots, treatment with $80 \mu \mathrm{M}$ 


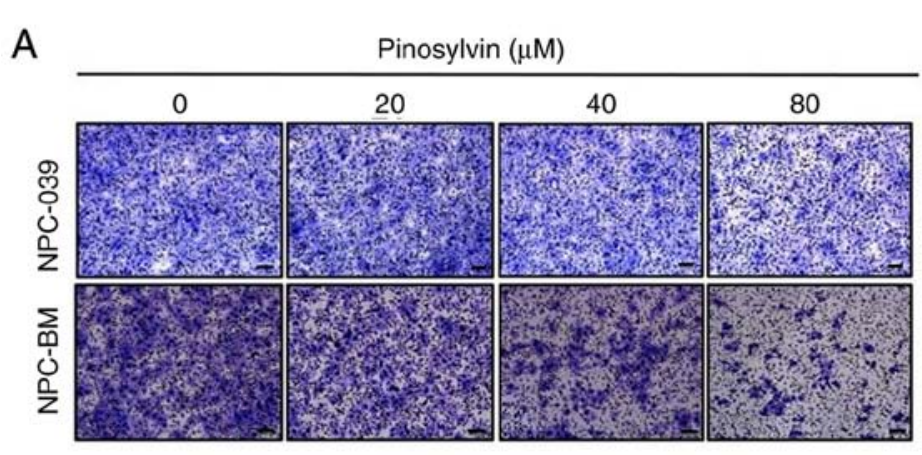

B

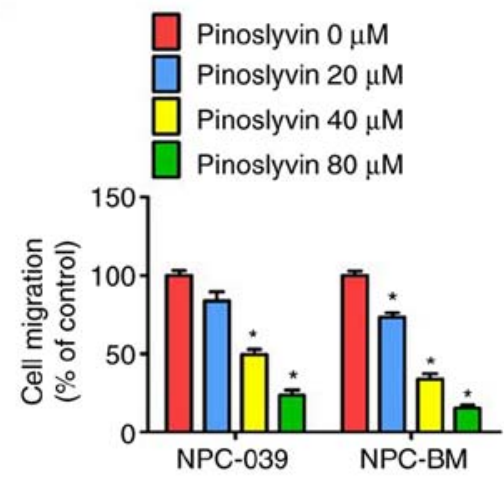

C
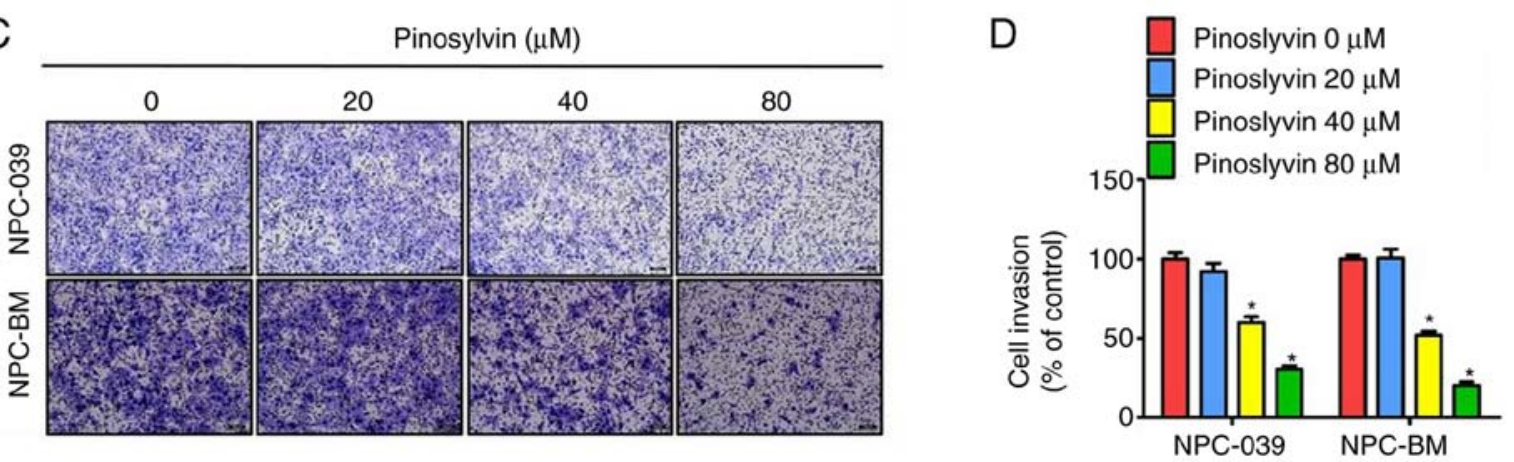

Figure 3. Pinosylvin inhibits migration and invasion in NPC cells. Transwell assay was performed to assess cell (A and B) migration and (C and D) invasion ability, which demonstrated a dose-dependent effect of pinosylvin on NPC cell migration and invasion ability. Data are presented as the mean \pm SD ( $=3$ ). ${ }^{*} \mathrm{P}<0.05$ vs. control. Scale bar, $100 \mu \mathrm{m}$. NPC, nasopharyngeal carcinoma.

pinosylvin decreased the phosphorylation of ERK1/2 and $\mathrm{p} 38$ to 63 and 51 in NPC-039 cells and 59 and 46\% in NPC-BM cells, respectively, at $24 \mathrm{~h}$ compared with untreated controls. By contrast, phosphorylation of JNK1/2 was significantly increased in the two NPC cell lines. In order to confirm the molecular mechanism underlying pinosylvin-induced inhibition of NPC cell migration, cells were pre-treated with specific inhibitors of ERK1/2, p38 and JNK1/2; following pre-treatment with specific inhibitors, pinosylvin-inhibited cell migration and invasion ability were significantly improved (Fig. 7A-D). Taken together, these findings indicate that pinosylvin exerted anti-metastatic effects via p38, ERK1/2 and JNK1/2 signaling pathways in human NPC cells.

\section{Discussion}

In an analysis of metastasis patterns of 629 patients with NPC, Huang et al (31) found that $95 \%$ of distant metastases occurred $<3$ years after completion of radiotherapy. Hence, determining effective methods of suppressing distant metastasis is important in the treatment of NPC. Plant polyphenols are important plant secondary metabolites with biological functions (such as countering infection by pathogens or mitigating environmental stresses), as well as antioxidant, anticancer and anti-inflammatory properties $(1,3,5,7)$. Studying compounds with such biochemical activity is beneficial for drug development in the pharmaceutical industry (32). Pinosylvin and resveratrol are terpenoid polyphenols with similar structures (2). Research has indicated that pinosylvin inhibits growth of human colorectal cancer cells (4), suppresses MMP-2 and MMP-9 activity in HT1080 cells (5) and suppresses migration and invasion in SCC-9, SAS and HSC-3 cell lines (27). In the present study, pinosylvin did not decrease the viability of the two NPC cell lines or a nasal cavity cancer cell line (RPMI 2650), however, high concentrations of pinosylvin inhibited the migration and invasion of NPC-039 and NPC-BM cells. Furthermore, the present results indicated that pinosylvin inhibited NPC cell metastatic effects by downregulating MMP-2/MMP-9 expression levels and modifying the regulation of EMT markers.

MMPs serve important roles in mediating cancer cell growth, differentiation, apoptosis, migration, invasion and angiogenesis (33). Di Carlo et al (34) performed zymography analysis and demonstrated that the ratio of MMP-9/MMP-2 in patients with cancer was increased compared with that in patients with benign disease and healthy individuals. High expression of MMP-2 and MMP-9 is significantly correlated with local and distant metastatic tumor recurrence and poor prognosis in head and neck squamous cell carcinoma (35-37). In the present study, gelatin zymography and western blotting were performed to analyze the effects of pinosylvin on MMPs in two NPC cell lines; pinosylvin significantly inhibited expression of MMP-2 and MMP-9 as well as MMP2 activity. Tissue inhibitors of metalloproteinase (TIMPs) control proteolytic activity and are a specific endogenous inhibitor of MMPs (38). Western blotting here showed that pinosylvin did not increase TIMP-1 or -2 protein levels in the two NPC cell lines (data not shown). This indicated that pinosylvin decreased MMP-2 protein expression levels and activity, via regulated the activation of zymogen at the post-transcriptional level. 
A

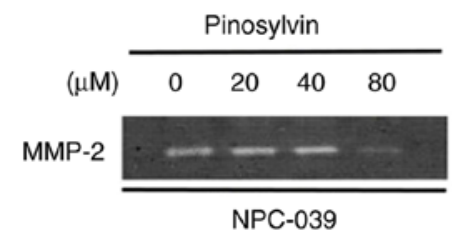

B

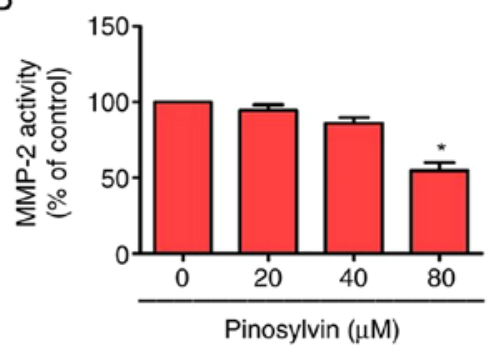

E

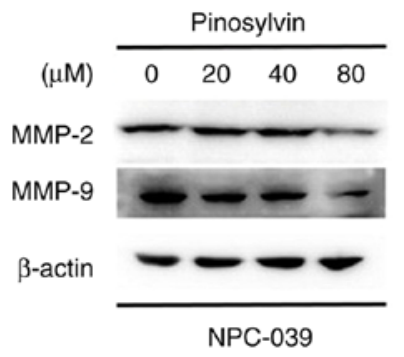

$\mathrm{F}$

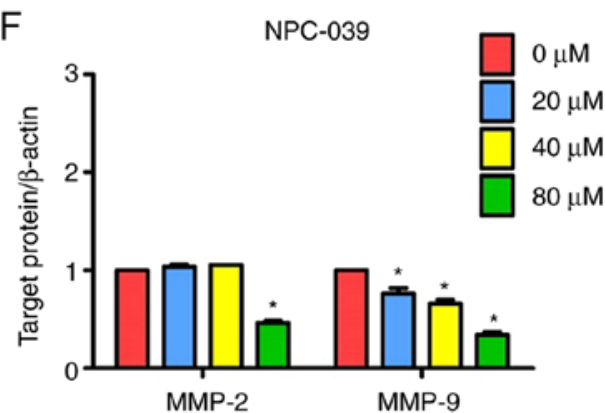

C

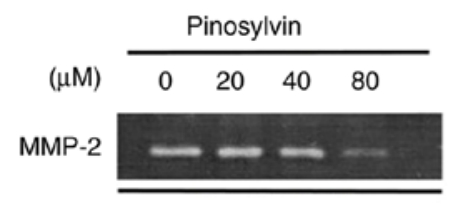

NPC-BM

D

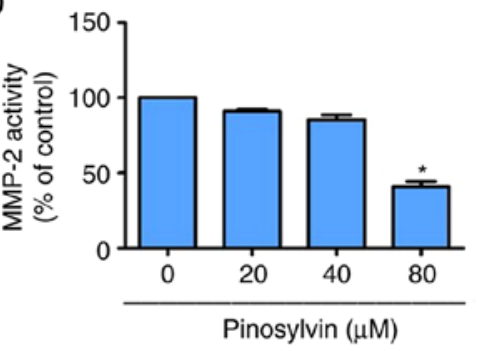

G

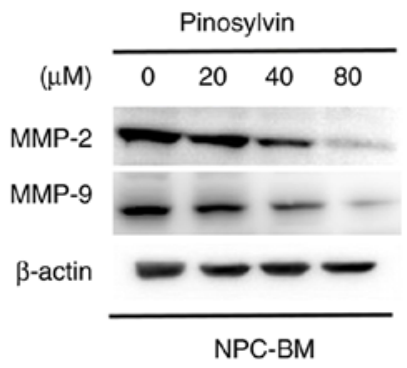

$\mathrm{H}$

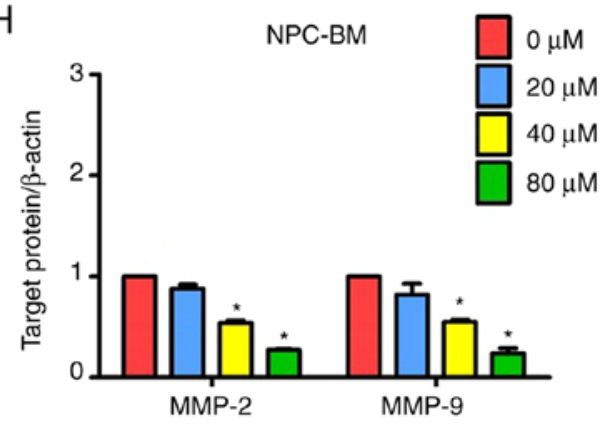

Figure 4. Effects of pinosylvin on activity of MMP enzymes and protein expression in NPC cells. Determination of MMP-2 enzyme activity using gelatin zymography in (A and B) NPC-039 and (C and D) NPC-BM cells. Western blotting was used to measure the expression of MMP-2 and MMP-9 protein after $24 \mathrm{~h}$ pinosylvin treatment in (E and F) NPC-039 and (G and H) NPC-BM cell lines. ImageJ software was used for quantitative analysis of protein. Data are presented as the mean $\pm \mathrm{SD}(\mathrm{n}=3)$. ${ }^{*} \mathrm{P}<0.05$ vs. control. NPC, nasopharyngeal carcinoma.

EMT is a key step in tumor cell migration and invasion in various types of human cancer (39-41). Upregulation of $\mathrm{N}$-cadherin induces EMT (40); another regulator of EMT is E-cadherin, which inhibits the occurrence of EMT and serves as a tumor suppressor (41). Vimentin is the primary cytoskeletal component of mesenchymal cells (42). ZO-1 and ZO-2 are required for tight junction formation and function $(43,44)$; mutations in ZO-1 and claudin-1 induce EMT (45). In the present study, pinosylvin-treated NPC-BM and NPC-039 cells exhibited significantly induced E-cadherin and ZO-1 expression, but decreased expression of $\mathrm{N}$-cadherin and vimentin. These findings suggest that pinosylvin inhibited EMT at the initiation step of tumor metastasis.

Compared with other intracellular signal transduction pathways (23), the MAPK pathway serves a more important role in cell proliferation, differentiation, apoptosis, angiogenesis and tumor metastasis $(23,24)$. A study indicated that TBL-12, a sea cucumber extract, inhibits migration and invasion of human PCa cells by inhibiting MMP-2 and MMP-9 via decreased phosphorylation of p38 (46). Additionally, 18 $\beta$-glycyrrhetinic acid inhibits migration and invasion of gastric cancer cells via the reactive oxygen species/protein kinase $\mathrm{C}-\alpha / \mathrm{ERK}$ signaling pathway (47). Therefore, the present study investigated whether the MAPK pathway is altered by pinosylvin treatment. Western blot analysis revealed that pinosylvin suppressed ERK1/2 and p38 protein phosphorylation but induced JNK protein phosphorylation in both NPC cell lines. This result is consistent with previously reported inhibition of Huh7 cell proliferation and metastasis by cucurbitacin E via suppression of MAPKs (48). A previous study showed that pinosylvin inhibits the growth of human colorectal cancer cells via suppression of Src/ERK and GSK-3/ $\beta$-catenin signaling (4). In our previous research, pinosylvin inhibited migration and invasion of oral cancer cells by suppressing the expression and activity of MMP-2 and ERK1/2 signaling (27). The present results suggest that pinosylvin was involved in MMP-2/MMP-9 regulation in NPC cells and that the MAPK pathway may serve a key role. 
A

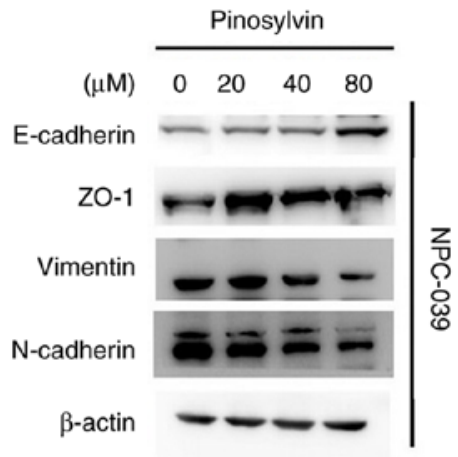

B

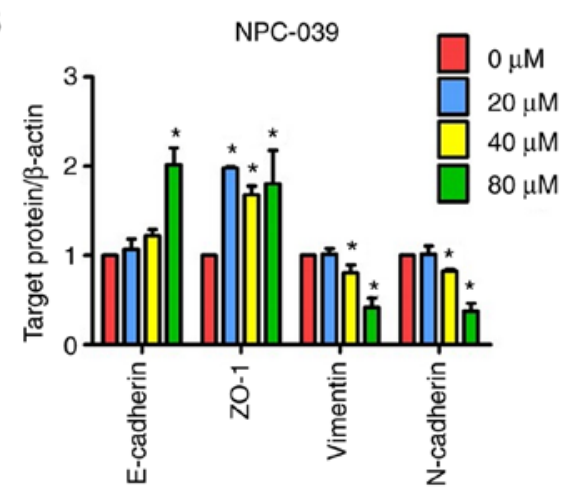

C

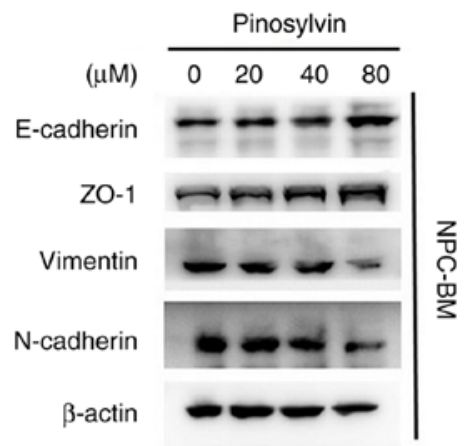

D

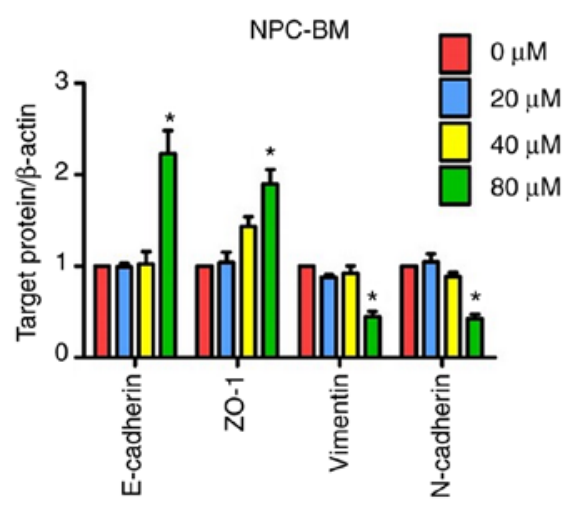

Figure 5. Pinosylvin affects mesenchymal marker protein expression in NPC cell lines. Western blotting was used to measure the expression of mesenchymal marker proteins following $24 \mathrm{~h}$ pinosylvin treatment in (A and B) NPC-039 and (C and D) NPC-BM cell lines. ImageJ software was used for quantitative analysis of protein. Data are presented as the mean $\pm \mathrm{SD}(\mathrm{n}=3)$. " $\mathrm{P}<0.05$ vs. control. NPC, nasopharyngeal carcinoma; ZO-1, zonula occludens-1.

A

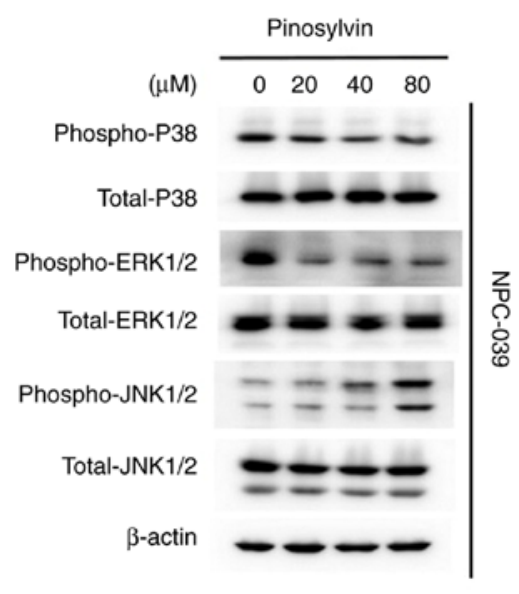

B

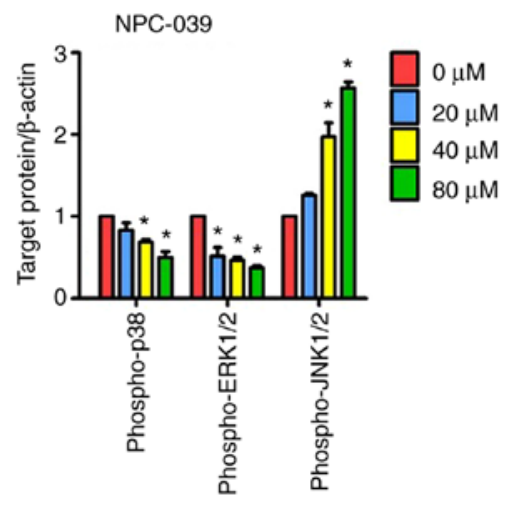

C

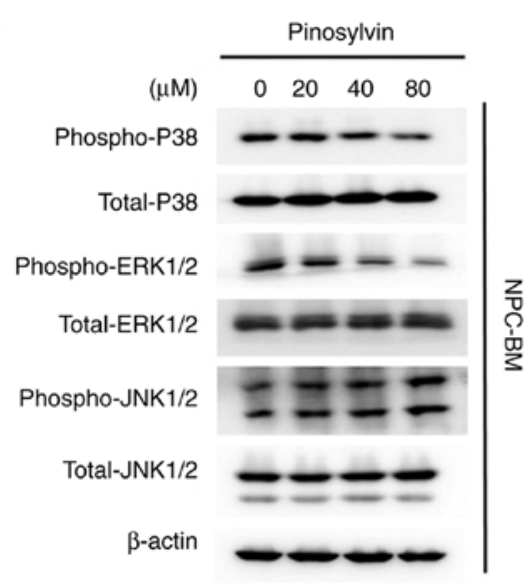

D

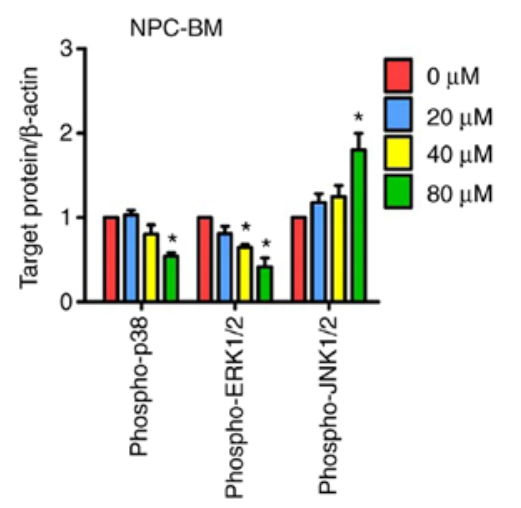

Figure 6. Pinosylvin affects MAPK pathways in NPC cell lines. Western blotting was used to measure expression levels changes in phospho-p38, -ERK1/2 and -JNK1/2 following $24 \mathrm{~h}$ pinosylvin treatment in (A and B) NPC-039 and (C and D) NPC-BM cell lines. Data are presented as the mean \pm SD ( $\mathrm{n}=3$ ). " $\mathrm{P}<0.05$ vs. control. NPC, nasopharyngeal carcinoma; phospho-, phosphorylated. 
A
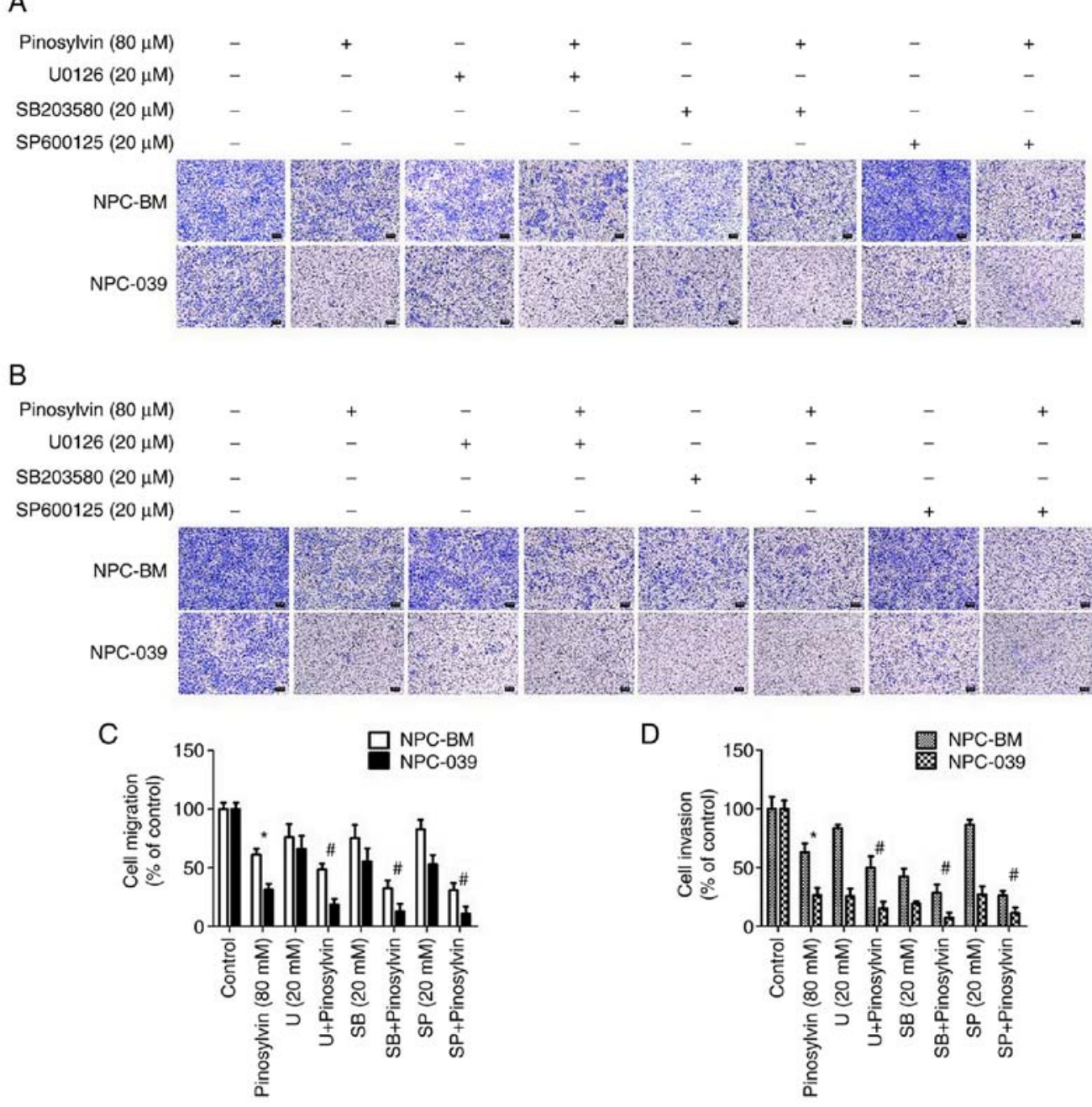

Figure 7. Pinosylvin inhibits migration and invasion by affecting the MAPK pathway in NPC cell lines. Cell lines were pre-treated with U, SB or SP for $1 \mathrm{~h}$, then with treated pinosylvin for $24 \mathrm{~h}$. Cell (A) migration and (B) invasion were determined via Transwell assay. Scale bar, $100 \mu \mathrm{m}$. Quantification of cell (C) migration and (D) invasion. Data are presented as the mean $\pm \mathrm{SD}(\mathrm{n}=3)$. ${ }^{*} \mathrm{P}<0.05$ vs. control; ${ }^{\mathrm{P}}<0.01$ vs. pinosylvin-alone. NPC, nasopharyngeal carcinoma; U, U0126; SB, SB20035820; SP, SP600125.

Identifying effective methods for treating distant metastases resulting from NPC is crucial. In summary, the present results demonstrated that pinosylvin decreased activity of MMP-2 and expression of MMP-2/MMP-9 in both NPC-BM and NPC-039 cell lines. Pinosylvin significantly inhibited both cell migration and invasion. The expression levels of epithelial markers increased, while those of mesenchymal markers decreased following treatment with pinosylvin. Following pre-treatment with specific inhibitors of ERK1/2, p38 and JNK1/2, pinosylvin-inhibited cell migration and invasion significantly improved. However, the lack of activator experiments is a potential limitation to the present study. A recent study suggested that pinosylvin is mostly metabolized in vivo and may provide a material basis for studying the pharmacological action of pinosylvin, thus providing information for the clinical treatment of chronic gastritis and gastric ulcers using Radix Linderae Reflexae (49). The short half-life and limited systemic exposure of pinosylvin prompt caution in its therapeutic application (50). However, the lack of in vivo experiments is a potential limitation to the present study. The present results suggested that pinosylvin may be useful in the development of drugs for treating NPC and preventing migration and invasion of NPC cells.

\section{Acknowledgements}

Not applicable.

\section{Funding}

The present study was supported by Changhua Christian Hospital (grant no. 109-CCH-IRP-013).

\section{Availability of data and materials}

All data generated or analyzed during this study are included in this published article.

\section{Authors' contributions}

$\mathrm{MCH}, \mathrm{MJH}$ and JTL conceptualized and designed the study. CCL, YCC, YSL and HYH. acquired, analyzed and interpreted data. $\mathrm{MCH}, \mathrm{YCC}$ and $\mathrm{MJH}$ drafted and revised the manuscript. 
MJH and JTL had overall responsibility for the published work. MJH and CCL confirm the authenticity of all the raw data. All authors read and approved the final manuscript.

\section{Ethics approval and consent to participate}

Not applicable.

\section{Patient consent for publication}

Not applicable.

\section{Competing interests}

The authors declare that they have no competing interests.

\section{References}

1. Riviere C, Pawlus AD and Merillon JM: Natural stilbenoids: Distribution in the plant kingdom and chemotaxonomic interes in vitaceae. Nat Prod Rep 29: 1317-1333, 2012.

2. Lee SK, Lee HJ, Min HY, Park EJ, Lee KM, Ahn YH, Cho YJ and Pyee JH: Antibacterial and antifungal activity of pinosylvin, a constituent of pine. Fitoterapia 76: 258-260, 2005

3. Koskela A, Reinisalo M, Hyttinen JM, Kaarniranta $\mathrm{K}$ and Karjalainen RO: Pinosylvin-mediated protection against oxidative stress in human retinal pigment epithelial cells. Mol Vis 20: 760-769, 2014

4. Park EJ, Chung HJ, Park HJ, Kim GD, Ahn YH and Lee SK Suppression of Src/ERK and GSK-3/ $\beta$-catenin signaling by pinosylvin inhibits the growth of human colorectal cancer cells. Food Chem Toxicol 55: 424-433, 2013

5. Park EJ, Park HJ, Chung HJ, Shin Y, Min HY, Hong JY, Kang YJ, Ahn YH, Pyee JH and Lee SK: Antimetastatic activity of pinosylvin, a natural stilbenoid, is associated with the suppression of matrix metalloproteinases. J Nutr Biochem 23: 946-952, 2012.

6. Torre LA, Bray F, Siegel RL, Ferlay J, Lortet-Tieulent J and Jemal A: Global cancer statistics, 2012. CA Cancer J Clin 65 87-108, 2015.

7. Chen AW, Tseng YS, Lin CC, His YT, Lo YS, Chuang YC, Lin SH, Yu CY, Hsieh MJ and Chen MK: Norcantharidin induce apoptosis in human nasopharyngeal carcinoma through caspase and mitochondrial pathway. Environ Toxicol 33: 343-350, 2018.

8. Chua MLK, Wee JTS, Hui EP and Chan ATC: Nasopharyngeal carcinoma. Lancet 387: 1012-1024, 2016.

9. Blanchard P, Lee A, Marguet S, Leclercq J, Ng WT, Ma J, Chan AT, Huang PY, Benhamou E, Zhu G, et al: Chemotherapy and radiotherapy in nasopharyngeal carcinoma: An update of the MAC-NPC meta-analysis. Lancet Oncol 16: 645-655, 2015.

10. Ng WT, Lee MC, Hung WM, Choi CW, Lee KC, Chan OS and Lee AW: Clinical outcomes and patterns of failure after intensity-modulated radiotherapy for nasopharyngeal carcinoma. Int J Radiat Oncol Biol Phys 79: 420-428, 2011.

11. Chaffer CL and Weinberg RA: A perspective on cancer cell metastasis. Science 331: 1559-1564, 2011.

12. Overall CM and Lopez-Otin C: Strategies for MMP inhibition in cancer: Innovations for the post-trial era. Nat Rev Cancer 2 : 657-672, 2002.

13. Egeblad M and Werb Z: New functions for the matrix metalloproteinases in cancer progression. Nat Rev Cancer 2: 161-174, 2002

14. Chien SY, Hsieh MJ, Chen CJ, Yang SF and Chen MK: Nobiletin inhibits invasion and migration of human nasopharyngeal carcinoma cell lines by involving ERK1/2 and transcriptional inhibition of MMP-2. Expert Opin Ther Targets 19: 307-320, 2015.

15. Sun YS, Thakur K, Hu F, Cespedes-Acuna CL, Zhang JG and Wei ZJ: Icariside II suppresses cervical cancer cell migration through JNK modulated matrix metalloproteinase-2/9 inhibition in vitro and in vivo. Biomed Pharmacother 125: 110013, 2020.

16. Webb AH, Gao BT, Goldsmith ZK, Irvine AS, Saleh N, Lee RP, Lendermon JB, Bheemreddy R, Zhang Q, Brennan RC, et al: Inhibition of MMP-2 and MMP-9 decreases cellular migration, and angiogenesis in in vitro models of retinoblastoma. BMC Cancer 17: 434, 2017.
17. Lyu Y, Xiao Q, Yin L, Yang L and He W: Potent delivery of an MMP inhibitor to the tumor microenvironment with thermosensitive liposomes for the suppression of metastasis and angiogenesis. Signal Transduct Target Ther 4: 26, 2019.

18. Huang W, Yan Y, Liu Y, Lin M, Ma J, Zhang W, Dai J, Li J, Guo Q, Chen $\mathrm{H}$, et al: Exosomes with low miR-34c-3p expression promote invasion and migration of non-small cell lung cancer by upregulating integrin $\alpha 2 \beta 1$. Signal Transduct Target Ther 5: 39, 2020.

19. Floor S, van Staveren WC, Larsimont D, Dumont JE and MaenhautC: Cancer cells in epithelial-to-mesenchymal transition and tumor-propagating-cancer stem cells: Distinct, overlapping or same populations. Oncogene 30: 4609-4621, 2011.

20. Wan FZ, Chen KH, Sun YC, Chen XC, Liang RB, Chen L and Zhu XD: Exosomes overexpressing miR-34c inhibit malignant behavior and reverse the radioresistance of nasopharyngeal carcinoma. J Transl Med 18: 12, 2020.

21. Lin K, Baritaki S, Militello L, Malaponte G, Bevelacqua Y and Bonavida B: The role of B-RAF mutations in melanoma and the induction of EMT via dysregulation of the NF-kappaB/Snail/RKIP/PTEN Circuit. Genes Cancer 1: 409-420, 2010.

22. Nakamura M and Tokura Y: Epithelial-mesenchymal transition in the skin. J Dermatol Sci 61: 7-13, 2011.

23. Guo YJ, Pan WW, Liu SB, Shen ZF, Xu Y and Hu LL: ERK/MAPK signalling pathway and tumorigenesis. Exp Ther Med 19: 1997-2007, 2020

24. Olea-Flores M, Zuñiga-Eulogio MD, Mendoza-Catalán MA, Rodríguez-Ruiz HA, Castañeda-Saucedo E, Ortuño-Pineda C, Padilla-BenavidesTandNavarro-TitoN:Extracellular-signalregulated kinase: A central molecule driving epithelial-mesenchymal transition in cancer. Int J Mol Sci 20: 2885, 2019.

25. Chen X, Xiao W, Liu X, Zeng M, Luo L, Wu M, Ye S and Liu Y: Blockade of Jagged/Notch pathway abrogates transforming growth factor $\beta 2$-induced epithelial-mesenchymal transition in human retinal pigment epithelium cells. Curr Mol Med 14: 523-534, 2014

26. Balogh P, Katz S and Kiss AL: The role of endocytic pathways in TGF- $\beta$ signaling. Pathol Oncol Res 19: 141-148, 2013.

27. Chen MK, Liu YT, Lin JT, Lin CC, Chuang YC, Lo YS, His YT and Hsieh MJ: Pinosylvin reduced migration and invasion of oral cancer carcinoma by regulating matrix metalloproteinase- 2 expression and extracellular signal-regulated kinase pathway. Biomed Pharmacother 117: 109160, 2019.

28. Yang SF, Chu SC, Liu SJ, Chen YC, Chang YZ and Hsieh YS: Antimetastatic activities of Selaginella tamariscina (Beauv.) on lung cancer cells in vitro and in vivo. J Ethnopharmacol 110: 483-489, 2007.

29. Ho HY, Lin CW, Chien MH, Reiter RJ, Su SC, Hsieh YH and Yang SF: Melatonin suppresses TPA-induced metastasis by downregulating matrix metalloproteinase-9 expression through JNK/SP-1 signaling in nasopharyngeal carcinoma. J Pineal Res 61: 479-492, 2016.

30. Nieto MA: Epithelial-mesenchymal transitions in development and disease: Old views and new perspectives. Int J Dev Biol 53: 1541-1547, 2009.

31. Huang CJ, Leung SW, Lian SL, Wang CJ, Fang FM and Ho YH: Patterns of distant metastases in nasopharyngeal carcinoma. Kaohsiung J Med Sci 12: 229-234, 1996.

32. Marienhagen J and Bott M: Metabolic engineering of microorganisms for the synthesis of plant natural products. J Biotechnol 163 : $166-178,2013$.

33. Rahimi Z, Yari K and Rahimi Z: Matrix metalloproteinase-9-1562T allele and its combination with MMP-2-735 $\mathrm{C}$ allele are risk factors for breast cancer. Asian Pac J Cancer Prev 16: 1175-1179, 2015.

34. Di Carlo A, Terracciano D, Mariano A and Macchia V: Matrix metalloproteinase-2 and matrix metalloproteinase-9 type IV collagenases in serum of patients with pleural effusions. Int J Oncol 26: 1363-1368, 2005.

35. Yoshizaki T, Maruyama Y, Sato H and Furukawa M: Expression of tissue inhibitor of matrix metalloproteinase-2 correlates with activation of matrix metalloproteinase- 2 and predicts poor prognosis in tongue squamous cell carcinoma. Int J Cancer 95: 44-50, 2001.

36. Riedel F, Gotte K, Schwalb J, Bergler W and Hormann K: Expression of 92-kDa type IV collagenase correlates with angiogenic markers and poor survival in head and neck squamous cell carcinoma. Int J Oncol 17: 1099-1105, 2000.

37. Hong SD, Hong SP, Lee JI and Lim CY: Expression of matrix metalloproteinase-2 and -9 in oral squamous cell carcinomas with regard to the metastatic potential. Oral Oncol 36: 207-213, 2000. 
38. Shrestha B, Bajracharya D, Byatnal AA, Kamath A and Radhakrishnan R: May High MMP-2 and TIMP-2 expressions increase or decrease the aggressivity of oral cancer? Pathol Oncol Res 23: 197-206, 2017.

39. Bagheri M, Fazli M, Saeednia S, Gholami Kharanagh $M$ and Ahmadiankia N: Sulforaphane modulates cell migration and expression of $\beta$-catenin and epithelial mesenchymal transition markers in breast cancer cells. Iran J Public Health 49: 77-85, 2020.

40. Lopez-Novoa JM and Nieto MA: Inflammation and EMT: An alliance towards organ fibrosis and cancer progression. EMBO Mol Med 1: 303-314, 2009.

41. Techasen A, Loilome W, Namwat N, Khuntikeo N, Puapairoj A, Jearanaikoon P, Saya H and Yongvanit P: Loss of E-cadherin promotes migration and invasion of cholangiocarcinoma cells and serves as a potential marker of metastasis. Tumour Biol 35: 8645-8652, 2014.

42. Chernoivanenko IS, Minin AA and Minin AA: Role of vimentin in cell migration. Ontogenez 44: 186-202, 2013 (In Russian).

43. Helfand BT, Chang L and Goldman RD: Intermediate filaments are dynamic and motile elements of cellular architecture. J Cell Sci 117: 133-141, 2004.

44. Shin K, Fogg VC and Margolis B: Tight junctions and cell polarity. Annu Rev Cell Dev Biol 22: 207-235, 2006.

45. Oliveira SS and Morgado-Diaz JA: Claudins: Multifunctional players in epithelial tight junctions and their role in cancer. Cell Mol Life Sci 64: 17-28, 2007.
46. Yuan L, Huang X, Zhou K, Zhu X, Huang B, Qiu S, Cao K and $\mathrm{Xu}$ L: Sea cucumber extract TBL-12 inhibits the proliferation, migration, and invasion of human prostate cancer cells through the p38 mitogen-activated protein kinase and intrinsic caspase apoptosis pathway. Prostate 79: 826-839, 2019.

47. Cai H, Chen X, Zhang J and Wang J: $18 \beta$-glycyrrhetinic acid inhibits migration and invasion of human gastric cancer cells via the ROS/PKC- $\alpha$ /ERK pathway. J Nat Med 72: 252-259, 2018.

48. Liu Y, Yang H, Guo Q, Liu T, Jiang Y, Zhao M, Zeng K and Tu P: Cucurbitacin $\mathrm{E}$ inhibits Huh7 hepatoma carcinoma cell proliferation and metastasis via suppressing MAPKs and JAK/STAT3 pathways. Molecules 25: 560, 2020.

49. Fu Y, Sun X, Wang L and Chen S: Pharmacokinetics and tissue distribution study of pinosylvin in rats by Ultra-High-Performance liquid chromatography coupled with linear trap quadrupole orbitrap mass spectrometry. Evid Based Complement Alternat Med 2018: 4181084, 2018.

50. Yeo SC, Luo W, Wu J, Ho PC and Lin HS: Quantification of pinosylvin in rat plasma by liquid chromatography-tandem mass spectrometry: Application to a pre-clinical pharmacokinetic study. J Chromatogr B Analyt Technol Biomed Life Sci 931: 68-74, 2013

This work is licensed under a Creative Commons Attribution-NonCommercial-NoDerivatives 4.0 International (CC BY-NC-ND 4.0) License. 